\title{
La loi sur le dossier électronique du patient: une opportunité
}

\section{Urs Stoffel}

Dr med., membre du Comité central de la FMH, responsable du département eHealth - infrastructure de sécurité et collecte des données

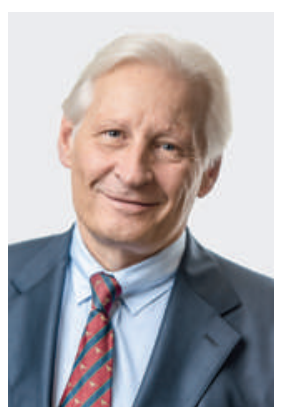

La loi fédérale sur le dossier électronique du patient (LDEIP) a été adoptée par le Parlement lors de la session d'été 2015. Le délai pour déposer un référendum a expiré et, selon le Conseil fédéral, la loi entrera en vigueur début 2017.

Dès le début, la FMH a analysé en détail la LDEIP afin de faire valoir de manière résolue et efficace le point de vue du corps médical tout au long du processus législatif et des échanges entre les deux Chambres avant l'adoption du projet définitif. Pour que les choses soient claires: la FMH approuve la loi adoptée. Nos deux exigences capitales concernant un numéro d'identification pour le dossier électronique du patient (DEP) indépendant du numéro AVS et le fameux caractère doublement facultatif pour les médecins installés et pour les patients ont été soutenues par le conseiller fédéral A. Berset. Après de nombreux hauts et bas, la loi finalement adoptée par le Parlement correspond dans une large mesure au message du Conseil fédéral.

\section{Le travail commence maintenant!}

Suite à l'adoption de la LDEIP, la FMH n'a aucune raison de se reposer sur ses lauriers et d'attendre les prochains événements. Elle entend veiller activement à ce que la mise en œuvre pratique de la loi apporte des avantages à nos membres. L'ordonnance de la LDEIP, qui est en cours d'élaboration, sera décisive pour savoir si les principales attentes en lien avec la loi pourront être réellement satisfaites.

\section{L'ordonnance de la LDEIP, qui est en cours} d'élaboration, sera décisive pour savoir si les principales attentes en lien avec la loi pourront être réellement satisfaites.

Le Groupe de travail interprofessionnel pour le dossier électronique du patient (GTI DEP) a été créé en 2014 par huit organisations professionnelles du domaine de la santé, en prévision de la mise en œuvre de la LDEIP et des travaux de l'organe de coordination eHealth Suisse. Toute proportion gardée, c'est une collaboration aussi exigeante qu'inédite, grâce à laquelle le groupe de tra- vail interprofessionnel fait preuve d'une nette avance par rapport à d'autres pays. Pour la FMH, il est extrêmement important que le corps médical, tout comme les autres associations professionnelles du domaine de la santé, soit pris au sérieux et participe activement à l'élaboration de l'ordonnance de la LDEIP. C'est le seul moyen de réussir l'implémentation du dossier électronique du patient et de le faire accepter aussi bien par la population que par les fournisseurs de prestations. Pour cela, la priorité doit d'une part toujours être mise sur les avantages pour les patients, mais d'autre part aussi pour les médecins.

\section{Les facteurs de réussite du DEP, du point de vue} des médecins du domaine ambulatoire:

- Règlementation claire concernant les recommandations de standards et de formats obligatoires et comment ils ont été adoptés.

- Encouragement et soutien des systèmes primaires pour la documentation électronique dans les cabinets des médecins installés, et en particulier des médecins de premier recours.

- Elaboration de recommandations visant à soutenir la mise en œuvre pratique de la cybersanté en général et du DEP en particulier.

- Elaboration commune des processus interprofessionnels dont découleront les contenus du DEP établis par le groupe de travail interprofessionnel.

- Garantie de la protection des données.

- Seules les données importantes pour le traitement doivent être consignées dans le DEP, afin d'éviter un "cimetière de données».

- Identification et traitement des interfaces, ou mieux des points de transit, qui éliminent les interruptions et présentent donc des avantages pour les patients et les médecins.

Avec son département eHealth - infrastructure de sécurité et collecte des données, la FMH est prête à collaborer étroitement avec tous les partenaires de la santé dans le but d'arriver ensemble à une mise en œuvre pertinente et efficace de la LDEIP. 